\title{
Funcionalidad familiar y automanejo de pacientes con diabetes mellitus e hipertensión arterial en el Hospital de Puente Piedra-Lima
}

\author{
Helen Lagos-Méndez ${ }^{1}$, Néstor Flores-Rodríguez ${ }^{2}$
}

\begin{abstract}
Lagos-Méndez H, Flores-Rodríguez N. Funcionalidad familiar y automanejo de pacientes con diabetes mellitus e hipertensión arterial en el Hospital de Puente Piedra-Lima. Cuid salud, jul-dic 2014; 1(2).
\end{abstract}

\section{RESUMEN}

La funcionalidad familiar y el automanejo son condiciones vitales que influyen en la calidad de vida de la persona que vive con alguna enfermedad crónica. Objetivo: determinar la relación entre la funcionalidad familiar y el automanejo de diabetes mellitus tipo 2 e hipertensión arterial en pacientes de 30-80 años, asistentes al consultorio de medicina interna en el hospital Carlos Lanfranco la Hoz de Puente Piedra-Lima. Metodología: estudio cuantitativo, diseño correlacional, de corte transversal, con una población de 100 pacientes con diabetes mellitus tipo 2 e hipertensión arterial; se emplearon 2 instrumentos: apgar familiar y automanejo en enfermedades crónicas. Resultados: muestran una correlación positiva entre la funcionalidad familiar y el automanejo (Rho de Spearman=0,43); además, el $54 \%$ de familias de los participantes evaluados fueron moderadamente disfuncionales y el $7 \%$ gravemente disfuncionales. El automanejo de los pacientes fue regular en un $56 \%$ e inadecuado en el $9 \%$. Conclusiones: la funcionalidad familiar y el automanejo son variables que se encuentran asociados de forma directa, lo cual influye en los patrones del comportamiento de las personas que viven con diabetes mellitus de tipo 2 y la hipertensión arterial.

Palabras clave: automanejo, funcionalidad familiar, diabetes mellitus, hipertensión arterial.
Lagos-Méndez H, Flores-Rodríguez N. Family functioning and self-management in patients with diabetes mellitus and hypertension in Puente Piedra Hospital-Lima. Cuid salud, jul-dic 2014; 1(2).

\begin{abstract}
Family functioning and self-management are vital conditions influencing the life quality of the person who live with any chronic disease. Objective: To determine the relationship between family functioning and self-management in patients 30 to 80 years with type 2 diabetes mellitus and hypertension attending consulting room of Internal Medicine Hospital Carlos Lanfranco $\mathrm{La} \mathrm{Hoz} \mathrm{of}$ Puente Piedra-Lima. Methodology: Quantitative approach correlational, cross-sectional design; with a population of 100 patients with type 2 Diabetes Mellitus and Hypertension; it was used two instruments: Family APGAR and selfmanagment in chronical disease. Results: It was identified positive correlation between family functioning and self-management (Rho de Spearman=0.43); besides, $54 \%$ of families of the participants are moderately dysfunctional families and $7 \%$ a severely dysfunctional families. For the self-management variable is obtained regular by $56 \%$, and inadequate in 9\%. Conclusion: Family functioning and self-management are variables directly associated, this influence the patterns of behavior of persons who lives with type 2 diabetes mellitus and hypertension.
\end{abstract}

Keywords: Self-management, family functioning, diabetes mellitus type 2, hypertension.

\footnotetext{
${ }^{1}$ Licenciada Enfermera. Egresada de la Escuela de Enfermería de la Universidad Cesar Vallejo.

${ }^{2}$ Magister en Educación, Licenciado Enfermero. Docente de la Escuela de Enfermería de la Universidad Cesar Vallejo.
} 


\section{INTRODUCCIÓN}

La Federación Internacional de Diabetes informa que la diabetes mellitus tipo 2 (DM2) y la hipertensión arterial (HTA), son enfermedades que afectan mundialmente a más de 371 millones de personas, causantes de la mortalidad de 4,8 millones del total de la población y, que la mayoría de casos se encuentra en los países en vías de desarrollo. ${ }^{1}$

En el Perú, la diabetes mellitus afecta a casi 2 millones de personas y es la décimo quinta causa de mortalidad. Así, en el 2012, según informe de la Oficina de Estadística e Informática del Ministerio de Salud existían en Lima 54638 pobladores afectados. $^{2}$ Ese mismo año, el Instituto Nacional de Estadística e Informática (INEI) reportó que el mayor porcentaje de personas con hipertensión arterial eran mujeres, alcanzando el $33,3 \%$ versus el 25,45\% en hombres, de un total de 1806000 adultos mayores. De este total, el 38,6\% presentó condición de pre-hipertensión y $34,4 \%$ hipertensión. ${ }^{3}$

La hiperglucemia crónica de la diabetes se asocia con daños a largo plazo, que provocan disfunción y fallas en varios órganos, en especial ojos, riñones, nervios, corazón y vasos sanguíneos, situación que se potencializa cuando existe también hipertensión arterial, dislipidemias y tabaquismo fundamentalmente. Estas complicaciones producen incremento de 3-4 veces de la morbimortalidad cardiovascular (macrovascular) y llegan a afectar notablemente la calidad de vida de los pacientes (microvascular y pie diabético), con elevados costes para el sistema sanitario. $^{4}$

Dichos daños pueden ser reducidos con abordaje integral de las personas, no solo controlando la hiperglicemia plasmática sino, al conjunto de factores que están presentes en estos pacientes, como son la hipertensión arterial, la dislipidemia, el tabaquismo, la obesidad, entre otros. ${ }^{4}$ Situación que amerita realizar cambios en los sistemas de salud, reforzando las medidas de prevención y promoción en salud, entre ellas las de educación en salud, comprometiendo a la comunidad en el cuidado de la salud, en la que estudiar la funcionalidad familiar y el automanejo de la enfermedad se constituyen en condiciones vitales importantes que contribuyen con la mejora de la calidad de vida de las personas.
A pesar de los avances logrados en el control de la enfermedad y sus complicaciones, el automanejo inadecuado de la enfermedad continúa siendo un problema serio a enfrentar, 5,6 resaltándose aquí la importancia del conocimiento sobre la enfermedad y el manejo terapéutico y emocional de la persona consigo misma. Propósito que podría ser alcanzado cuando existe un trabajo colaborativo entre paciente, familia y profesional de la salud, orientado a enseñar, acompañar y supervisar el proceso de salud-enfermedad; en la que las decisiones del tratamiento sean tomadas bajo un compromiso de co-responsabilidad y con pacientes bien informados.

La Organización Mundial de la Salud (OMS) reconoce que en las enfermedades crónicas, el paciente y su familia son elementos claves, quienes deben estar preparados, informados y motivados, con el desarrollo de suficientes habilidades para el automanejo de la enfermedad. La efectividad del automanejo ayuda al paciente y familia adherir al tratamiento minimizando las complicaciones, síntomas y problemas crónicos asociados a incapacidades. ${ }^{7}$ Campo y Portillo ${ }^{5}$ resaltan que la familia es un soporte importante para el automanejo, pero es necesario que tanto ellos como el paciente estén suficientemente informados sobre la enfermedad y necesidades de cuidado, para así constituirse (la familia) en entes de soporte facilitadores y no estresores.

Un estudio realizado en Perú ${ }^{6}$ reporta que el automanejo es deficiente, con una media general de 66,1 puntos (de 96) para diabetes, hipertensión y cáncer. En general, el automanejo en las enfermedades crónicas fue deficiente en todas las dimensiones: conocimiento, adherencia y manejo de signos y síntomas. Por otro lado, estudios realizados en diversos países reportan que existe la necesidad que los pacientes se encuentren debidamente informados sobre sus propios cuidados y tratamiento, información que debe trasmitirse en lenguaje comprensible y claro. ${ }^{5}$

Bajo ese contexto, este estudio pretende determinar si existe relación entre la funcionalidad familiar y el automanejo, como condiciones básicas en el afrontamiento para prevenir, rehabilitar y reducir las complicaciones de la diabetes e hipertensión arterial en pacientes de 30-80 años de edad, con el fin de mantener un cuidado optimo, generando estilos de vida saludables. 


\section{METODOLOGÍA}

El estudio fue de enfoque cuantitativo, método observacional, diseño descriptivo correlacional de corte transversal; considerando las variables en estudio: funcionalidad familiar y el automanejo. Fue realizado entre abril y julio del 2014, en el servicio de Medicina Interna del Hospital Carlos Lanfranco La Hoz, ubicado en el distrito de Puente Piedra-Lima/Perú. El muestreo utilizado fue no probabilístico por conveniencia y la muestra estuvo constituida por los 100 pacientes que acudieron a consultorio durante los meses del estudio; cuyas edades fluctuaban entre 30-80 años, de ambos sexos, con diagnóstico médico de diabetes mellitus tipo 2 e hipertensión arterial con un tiempo de enfermedad mayor a un año.

La técnica utilizada fue la encuesta y como instrumentos dos cuestionarios, para la funcionalidad familiar el Apgar Familiar y para automanejo el de Automanejo en Enfermedades Crónicas.

El Apgar Familiar fue diseñado en 1978 por el Dr. Gabriel Smilkstein (Universidad de Washington), validado en España $^{8}$ en 656 pacientes obteniéndose un alfa de Crombach de 0,84 , siendo los coeficientes de correlación intraclase de sus 5 ítems superiores a 0,55. Asimismo, este instrumento fue validado el 2006 en Colombia ${ }^{9}$ con estudiantes de educación secundaria obteniéndose un valor de alfa de Cronbach de 0,793.

Con Respecto al instrumento Automanejo en Enfermedades Crónicas, fue validado en LimaPerú el 2012 en una población circunscrita a los centros de salud del primer nivel de atención del Ministerio de Salud, en personas con enfermedades crónicas (diabetes, cáncer o hipertensión arterial), obteniéndose como resultado un alfa de Cronbach de 0,89, con un análisis factorial que explica el $58 \%$ de varianza e identifica tres factores: adherencia, conocimientos y manejo de efectos secundarios, sin modificar el número y contenido de las preguntas originales. ${ }^{10}$

En el estudio ambas variables han sido dimensionadas de la siguiente manera: para Funcionalidad familiar se consideran las dimensiones: adaptabilidad, participación, crecimiento, afecto y resolución; mientras que para el Automanejo: adherencia al tratamiento, manejo de los síntomas y conocimiento de la enfermedad.
Con relación a las técnicas de procesamiento y análisis de datos, se utilizó el programa SPSS v.21 (Statistical Package Of Social Science). Previamente, los datos fueron digitados en programa Excel 2010 utilizando códigos que mantuvieron el anonimato de los encuestados. Para la presentación de los resultados se utilizan tablas y/o gráficos para su mejor interpretación. También se hizo uso de la estadística descriptiva e inferencial, para lo cual se aplicó la prueba chi cuadrado y correlación de Spearman, con el fin de establecer relación de variables considerando significativo un $\mathrm{p}<0,05$.

El proyecto fue revisado y aprobado por el comité de ética en investigación de la Universidad Cesar Vallejo, resaltando que se respetaron en todo momento los principios bioéticos como la autonomía, beneficencia, no maleficencia y justicia.

\section{RESULTADOS}

Los resultados evidencian que la mayoría de pacientes pertenecía al género femenino (54\%), cuyas edades fluctúan entre 30-59 (46\%) y 60-80 (54\%), con mayor cantidad de personas con estudios secundarios (47\%) y cuyo tiempo de enfermedad oscilaba predominantemente entre 610 años $(52 \%)$.

Gráfico 1: Funcionalidad familiar de pacientes con diabetes mellitus tipo 2 e hipertensión arterial. Consultorio de Medicina Interna, HCLLH 2014.

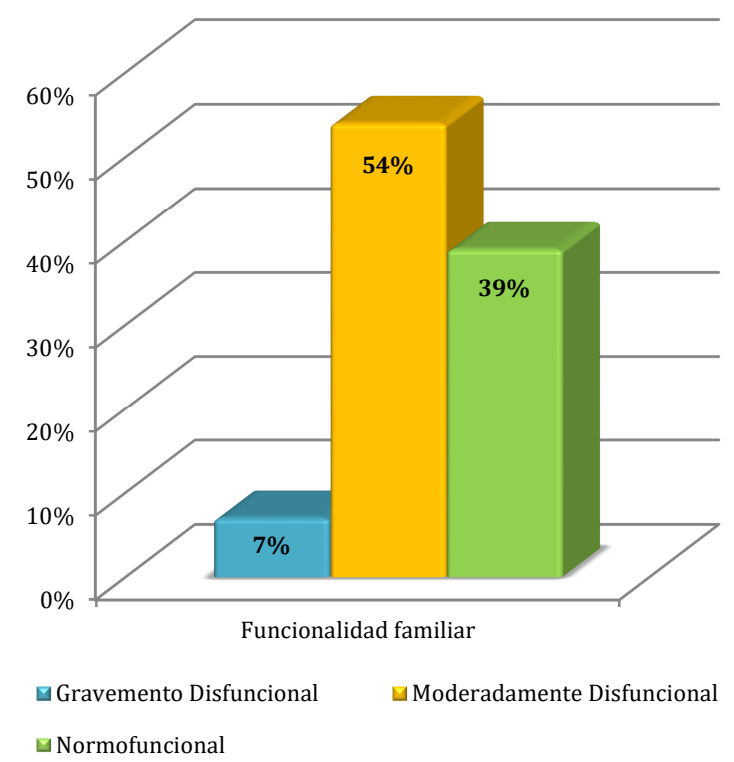

En el gráfico 1, se observa que el $54 \%$ de pacientes conforma familias moderadamente disfuncionales y el $7 \%$ a familias gravemente disfuncionales. 
Gráfico 2: Automanejo de pacientes con diabetes mellitus tipo 2 e hipertensión arterial. Consultorio de Medicina Interna, HCLLH 2014.

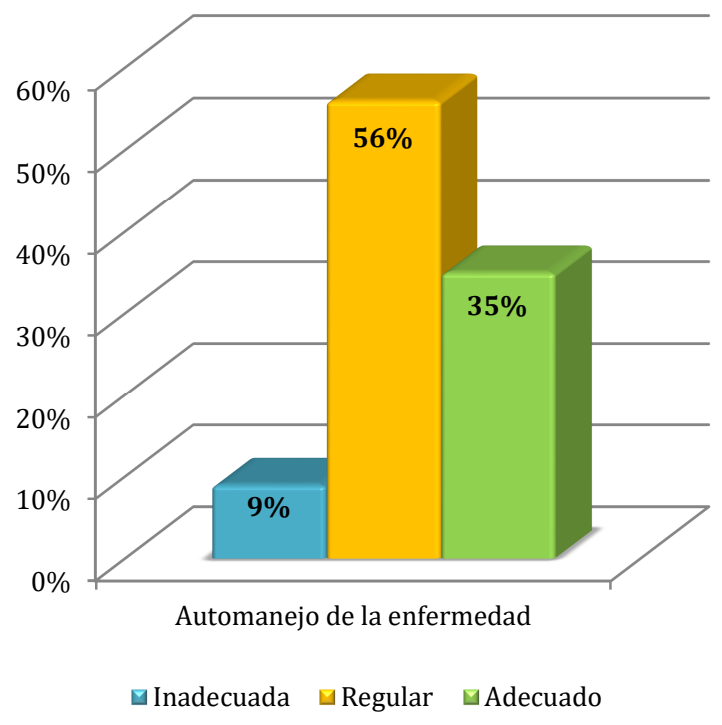

En el gráfico 2, respecto al automanejo de sus enfermedades por los pacientes, predominó el automanejo regular (56\%), observándose además en el $9 \%$ automanejo inadecuado.

Gráfico 3: Automanejo según dimensiones en pacientes con diabetes mellitus tipo 2 e hipertensión arterial. Consultorio de Medicina Interna, HCLLH 2014.

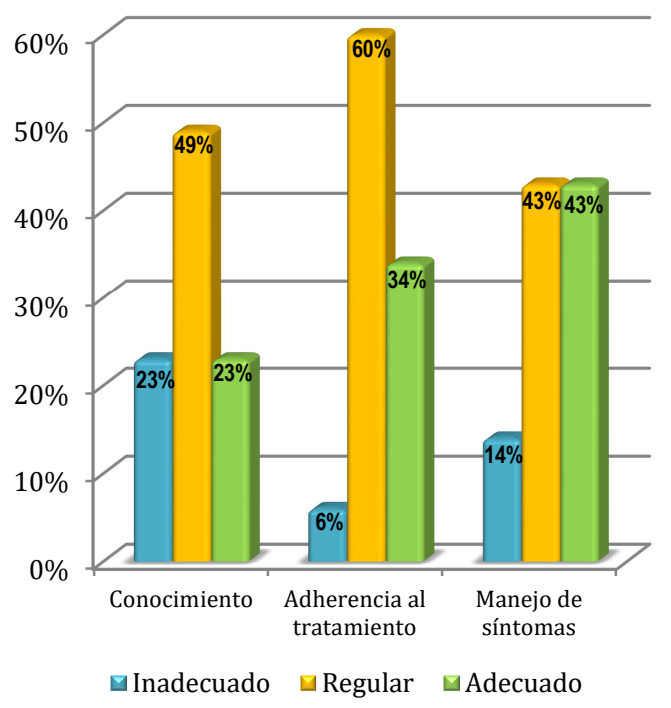

En el gráfico 3, automanejo según dimensiones, en general, se observa que predominó la categoría regular en todas: conocimiento $(49 \%)$, adherencia al tratamiento (60\%) y manejo de los síntomas (43\%), aunque en este último un igual porcentaje de pacientes presentó automanejo adecuado.
Gráfico 4: Funcionalidad familiar y automanejo en pacientes con diabetes mellitus tipo 2 e hipertensión arterial. Consultorio de Medicina Interna del HCLLH 2014.

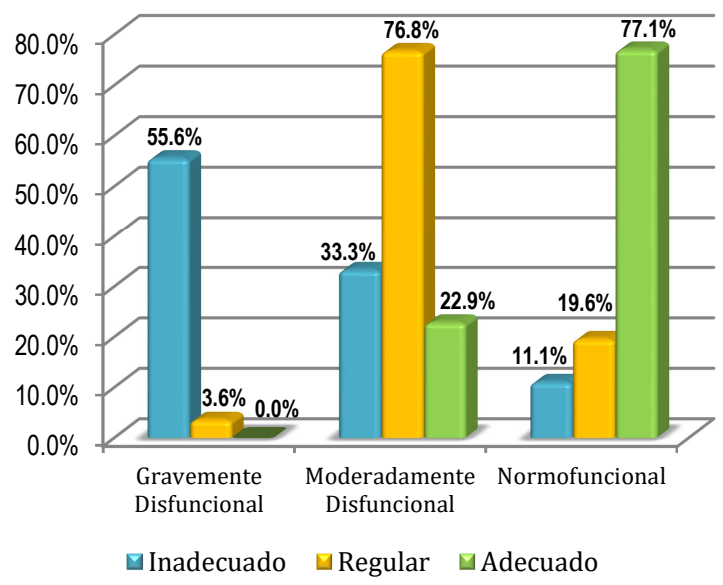

Finalmente, en el gráfico 4 respecto al análisis bivariado, se observa que el paciente que procede de una familia gravemente disfuncional presentó un automanejo inadecuado $(55,6 \%)$; a su vez, en pacientes con familias moderadamente disfuncionales el automanejo fue regular $(76,8 \%)$ $\mathrm{y}$, en el caso de pacientes con familias normofuncionales predominó el automanejo adecuado $(77,1 \%)$. Siendo que la prueba de chicuadrado mostró un valor de $\mathrm{p}=0,000$. Asimismo, la prueba de Rho de Spearman identificó una correlación de 0,543 con un valor de $\mathrm{p}=0,000$ determinando una correlación positiva entre variables

\section{DISCUSIÓN}

Familias funcionales son aquellas capaces de crear un entorno que facilite el desarrollo personal de sus miembros y en general, no sufren crisis ni trastornos psicológicos graves. ${ }^{11}$

La funcionalidad aparece asociada a diferentes características del sistema familiar como los niveles de cohesión, flexibilidad, comunicación, estrés, conflictos, vínculos, entre otros. ${ }^{11}$ Según McDaniel et al., citado por Concha y Rodríguez, ${ }^{12}$ la familia puede contribuir con la salud o enfermedad de sus miembros, mediante el desempeño de sus actividades, como por ejemplo, con la presencia o control de una patología crónica en uno o varios de sus integrantes. Los autores resaltan que existe alta concurrencia de riesgo cardiovascular en las familias, por el mismo hecho de que la familia comparte los mismos factores de riesgo; en tal sentido, conocer la dinámica familiar en su contexto es relevante, dado que las alteraciones de la vida familiar son capaces de 
provocar desequilibrio emocional y descompensación en el estado de salud de la persona. Especialmente en las enfermedades crónicas como la diabetes y la hipertensión arterial, en las que la evolución y respuesta al tratamiento depende, en buena medida, del funcionamiento familiar.

La comunicación efectiva, la efectividad, el desarrollo, la adaptabilidad, el apoyo emocional, económico y de información, son funciones esenciales que tienen efecto positivo en la recuperación de la salud. Por lo tanto, familias que no cumplen estas funciones o no saben manejar el estrés que produce la enfermedad, influirán en la evolución negativa de la misma. ${ }^{12}$

Los resultados del estudio muestran que los pacientes pertenecen a familias moderadamente disfuncionales, seguido de las normofuncionales (gráfico 1). Estos datos se correlacionan con el estudio realizado en un hospital de tercer nivel en Nigeria, respecto al apoyo que el paciente recibe de su familia mostró que el $51 \%$ tenía un débil apoyo, seguido de un apoyo fuerte y por último aquellos que no tenían apoyo. ${ }^{13}$

En los pacientes con enfermedades crónicas, la funcionalidad familiar constituye un soporte fundamental para apoyarlo en situaciones de déficit, de debilidad y de angustia. Aquí el familiar viene a ser un complemento que da fuerza, conocimiento y voluntad como lo menciona Henderson. ${ }^{14}$ Cuando la familia y el paciente están conscientes de la enfermedad, su control y sus riesgos tienen independencia para poder afrontar situaciones propias de la enfermedad, que muchas veces desgasta emocionalmente a la persona y a la familia como un todo. El estudio de Nigeria demostró que a menor apoyo de la familia mayores niveles de hemoglobina glucosilada en los pacientes. $^{13}$

$\mathrm{Al}$ evaluar el automanejo de la enfermedad se observa una mayor frecuencia de automanejo regular, seguido de adecuado e inadecuado (gráfico 2), demostrando que las prácticas de cuidado a la salud en la vida diaria no son del todo saludables. El estudio de Luna y Sedeno ${ }^{15}$ puede relacionarse con estos resultados, dado que evaluaron adherencia y apoyo familiar en pacientes con diabetes mellitus, habiéndose identificado una óptima adherencia, seguido de adherencia regular. Resultados contrarios a los obtenidos por Peñarrieta-De-Córdova et al., ${ }^{6}$ quienes hallaron automanejo deficiente en la población de dos centros del Ministerio de Salud del Perú.

Según la OMS, ${ }^{16}$ la adherencia a la terapéutica incluye mucho más que tomar el medicamento y cumplir con las consultas de seguimiento; deben realizarse paralelamente importantes modificaciones en los comportamientos relacionados con la higiene personal, el autocuidado y la incorporación de estilos de vida saludables para prevenir riesgos, como una alimentación adecuada, ejercicio físico regular y abandono de hábitos nocivos. En ese sentido, la evaluación del automanejo por dimensiones, muestra que en conocimiento y adherencia al tratamiento predominó la categoría regular y en manejo de síntomas la categoría regular y adecuada (gráfico 3). Estos resultados difieren Peñarrieta-De-Córdova et al., ${ }^{6}$ quienes reportaron que el automanejo en personas con enfermedades crónicas es deficiente en todas sus dimensiones: conocimiento, adherencia, y manejo de signos y síntomas.

Datos de la OMS $^{16}$ muestran que la adherencia a la terapéutica en enfermedades de largo plazo es de $50 \%$ en la población en general, cifra que es menor en los países en desarrollo por las carencias presentes en los sistemas de salud y sus modelos de atención; además de los múltiples factores que influyen en la misma, entre ellos los factores intrapersonales como la edad, sexo, autoestima, autoefectividad, estrés, depresión y consumo de alcohol; los factores interpersonales como la relación paciente-profesional de salud y el apoyo social y, los factores ambientales, resaltando los económicos, agrícolas, políticos, de atención de salud, geográficos, ecológicos y culturales.

La correlación de las variables en estudio muestra que los pacientes que conforman familias gravemente disfuncionales tienen mayor predisposición a presentar un automanejo inadecuado, siendo que en las moderadamente disfuncionales el automanejo es regular (gráfico 4). En el estudio de Bail y Azzollini, ${ }^{17}$ la mayoría de pacientes con actitud positiva mencionó que su familia siempre los apoyaba. Otro estudio realizado en el país en un hospital de tercer nivel concluye que los pacientes que pertenecen a familias normofuncionales presentaron un adecuado control glucémico en comparación con aquellos que tuvieron disfunción leve y grave. ${ }^{18}$ Concha y Rodríguez $^{12}$ relacionando la funcionalidad familiar con la compensación y descompensación de la enfermedad en pacientes de 
un Centro de Salud en Chillón-Chile, identificaron que existe mayor susceptibilidad de descompensación en el paciente cuando la familia tiene una funcionalidad moderada o disfunción severa. Sin embargo, no siempre existe esta correlación; Jimenez-Benavides et al., ${ }^{19}$ identificaron que la disfunción familiar tiene poca importancia en el control de la glucosa en pacientes con diabetes tipo 2 , pues el $46,3 \%$ de la población estudiada presentó una función familiar adecuada pero con elevados valores de glucosa en sangre.

Sobre el automanejo en la hipertensión arterial, es importante considerar la percepción y conocimiento que el paciente tiene sobre la enfermedad y la habilidad para controlar los síntomas; siendo que monoterapias con régimen sencillo de dosificación suelen favorecer el control del proceso salud-enfermedad. ${ }^{16}$ En el estudio fueron considerados pacientes con diabetes $\mathrm{e}$ hipertensión arterial, lo que denota la complejidad de la terapia medicamentosa y riesgo elevado para desarrollar enfermedades macrovasculares, requiriendo que la familia ejerza un gran soporte para sobrellevar y controlar las patologías subyacentes.

La $\mathrm{OMS}^{16}$ resalta que la adherencia terapéutica (o automanejo) es compleja en pacientes con enfermedades crónicas, debido a la interferencia de múltiples factores, entre los que se destaca el ambiental, pues en la vida actual se han generado muchos cambios, sobre todo, económicos y políticos que impactan fuertemente en el modo de vivir de las personas en todo el mundo como: el acceso a comidas rápidas a bajo costo, el mayor sedentarismo propios de los sistemas y modos de trabajo, el crecimiento de las ciudades y la necesidad de medios de transporte, la mayor inserción de la mujer en la producción económica, que han generado nuevos modelos de

\section{Correspondencia:}

Néstor Flores Rodríguez

Correo electrónico: nestorfr@ hotmail.com

\section{REFERENCIAS BIBLIOGRÁFICAS}

1. DF: International Diabetes Federation. Diabetes Atlas. $5^{\mathrm{a}}$ edition 2012 update [internet]. Brussels-Belgium: IDF; 2012, [citado 05 de abr 2013]. [aproximadamente 06 laudas]. Disponible en: http://www.indiaenvironmentportal.org.in/file s/file/diabetes\%atlas\%2012.pdf familia, la aculturización de las prácticas cotidianas incorporando modelos que muchas veces colisionan con la identidad nacional y etnica, las prioridades en la atención de salud, la mayor demanda por una atención de calidad, el menor acceso al sistema de salud, entre otros. Todos estos aspectos y muchos otros de carácter personal y de relación con el sistema de salud, deben ser considerados al analizar cómo los pacientes con enfermedad crónica pueden estar manejando sus procesos de salud-enfermedad y en qué medida influye la dinámica familiar.

El estudio presenta algunas limitaciones que deben ser consideradas al evaluar sus resultados, como su diseño transversal, el muestreo no probabilístico, su tamaño muestral, que no necesariamente comprende una población de base. Debiendo considerarse también que se presenta un análisis global de los datos y no por grupos etarios, nivel de escolaridad, tiempo de enfermedad y patologías decurrentes de la diabetes e hipertensión arterial.

Concluyendo, los resultados muestran correlación significativa positiva entre las variables, es decir, cuanto mayor o menor sea la funcionalidad familiar será mayor o menor el automanejo de la enfermedad crónica. Resaltando que en futuros estudios de funcionalidad familiar deberá incluirse análisis por grupos etarios de adultos y adultos mayores, además de nivel de escolaridad y tiempo de enfermedad, con el fin de obtener datos más precisos. Por otro lado, el personal de salud debe trabajar colaborativamente con el paciente, valorando la importancia del buen automanejo de la enfermedad, en la que este último asume un rol activo, flexible y responsable en su autocuidado, en lugar de limitarse a seguir reglas rígidamente prescritas; solo de ese modo se evitarán las complicaciones que terminan por afectar su calidad de vida y la de su familia.
2. Ministerio de Salud. Especial de Diabetes [homepage de internet]. Lima; 2010, [actualizado SN; citado 05 de abr 2013]. Disponible en: http://www.minsa.gob.pe/portada/Especiales/2 010/diabetes/datos.asp 
3. INEI: Instituto Nacional de Estadística e Informática. Estado de la población peruana 2013. Población adulta mayor [internet]. Lima; INEI; 2013 [citado 05 de abril 2013]. 40p. Disponible en: http://www.inei.gob.pe/media/MenuRecursivo /publicaciones_digitales/Est/Lib1095/libro.pdf

4. Medaviella BJJ. Complicaciones de la diabetes. Diagnóstico y tratamiento. SEMERGEN [serie de internet]. 2001 [citado 15 de mar 2015]; 127: 132-45. Disponible en: http://apps.elsevier.es/watermark/ctl_servlet? $\underline{\mathrm{f}=10 \text { pident articulo }=10022281 \text { pident_usuario }}$ $=0$ pcontactid $=$ pident_revista $=40 \&$ ty $=142$ accio $\underline{\mathrm{n}=\text { L\&origen }=\text { zonadelecturawe } \mathrm{b}=\mathrm{www} \text {.elsevie }}$ $\underline{\text { r.eslan }=e s f i c h e r o}=40 \mathrm{v} 27 \mathrm{n} 03 \mathrm{a} 10022281 \mathrm{pdf001}$ .pdf

5. Campo GC, Portillo MC. El automanejo de los pacientes con diabetes tipo 2: una revisión narrativa. An Sist Sanit Navar [serie de internet]. Sep-dic 2013 [citado 20 de feb 2015]; 36(3): 489-510. Disponible en: http://scielo.isciii.es/pdf/asisna/v36n3/14_revi siones.pdf

6. Peñarrieta-De-Córdova $\mathrm{M}$, Vergel-Camacho S, Lezama-Vigo S, Rivero- Álvarez R, TaipeCancho J, Borda-Olivas H. El automanejo de enfermedades crónicas: población de una jurisdicción de centros de salud. Rev enferm Herediana [serie de internet]. 2013 [citado 10 feb 2015]; 6(1):42-49. Sitio: http://www.upch.edu.pe/vrinve/dugic/revistas/ index.php/RENH/article/view/2376/2333

7. WHO: World Health Organization. Innovate caer for chronic conditions: building Blocks for Action [internet]. Geneva: WHO; 2002 [citado 10 de mar 2015]. 103 p. Disponible en: http://www.who.int/chp/knowledge/publicatio ns/icccglobalreport.pdf

8. Bellón JA, Delgado A, Luna JD, Lardelli P. Validez y fiabilidad del cuestionario de función familiar Apgar-familiar. Aten Primaria [serie de internet]. 1996 [citado 10 de enero 2014], 18(6): 289-295. Disponible en: http://www.elsevier.es/es-revista-atencionprimaria-27-articulo-validez-fiabilidad-delcuestionario-funcion-14357

9. Forero ALM, Avendaño DMC, Duarte CZJ, Campo-Arias A. Consistencia interna y análisis de factores de la escala APGAR para evaluar el funcionamiento familiar en estudiantes de básica secundaria. Revista Colombiana de Psiquiatría [serie de Internet]. Mar 2006 [citado 10 de ene 2014], XXXV (1): 23-9. Disponible en: http://www.scielo.org.co/pdf/rcp/v35n1/v35n1 a03.pdf

10. Peñarrieta $\mathrm{CM}$, Vergel $\mathrm{CO}$, Schmith $\mathrm{AL}$, Lezama VS, Rivero AR, Taipe CJ, Bejarano BLW. Validación de un instrumento para evaluar el automanejo en enfermedades crónicas en el primer nivel de atención en salud. Rev Cient de Enferm [serie de internet]. 2012 [citado 12 de dic 2013], VIII (1):64-73. Disponible en: http://www.cep.org.pe/cn/revista3/JOB16352 13 Revista_Cientifica.pdf

11. Gonzáles FS, Gimeno CA, Meléndez MJC, Córdova IA. La percepción de La funcionalidad familiar. Confirmación de su estructura bifactorial. Escitos de Psicología [serie de internet]. 2010 [citado 10 de ene 2015]; 5(1): 34-9. Disponible en: http://scielo.isciii.es/pdf/ep/v5n1/informe4.pdf

12. Concha TMC, Rodríguez GCR. Funcionalidad familiar en pacientes diabéticos e hipertensos compensados y descompensados. Theoria [serie de internet]. 2010. [citado 06 de abr 2013]; 19(1):41-50. Disponible en: http://www.ubiobio.cl/miweb/webfile/media/1 94/v/v19-1/3.pdf

13. Adetunji AA. Ladipo MMA. Irabor AE y Adeleye JO. Perceived family support and blood glucose control in type 2 diabetes. Mera: Diabetes Internacional [serie de internet]. Sep 2007 [citado 26 de may 2013]; 18,19. Disponible en: http://indexmedicus.afro.who.int/iah/fulltext/D IJ/nov07/DI20Nov20072018-19.pdf

14. Tomey MA. Virginia Henderson. Definición de la enfermería clínica. In: Tomey MA, Alligood RM. Modelos y teorías de enfermería. 5ta. Ed. Elservier: Madrid-España, 2005. Pp.98111.

15. Luna C, Sedeno Y. Apoyo familiar y adherencia al tratamiento en usuarios adultos con diabetes mellitus tipo 2 del consultorio externo de endocrinología del HNDAC del Callao [tesis de bachiller]. Lima: Universidad César Vallejo; 2012. 
16. OMS: Organización Mundial de la Salud. Adherencia a los tratamientos a largo plazo. Pruebas para la acción [internet]. Washington, DC: OMS (Unidad de Enfermedades no trasmisibles); 2004 [citado 10 de mar 2015]. 202p. Disponible en: http://www.paho.org/hq/index.php?option=co $\underline{\mathrm{m} \text { docman } \& \text { task }=\text { doc } \text { view } \& \text { gid }=18722 \& \text { Ite }}$ $\underline{\text { mid }}=$

17. Adetunji AA. Ladipo MMA. Irabor $\mathrm{AE} y$ Adeleye JO. Perceived family support and blood glucose control in type 2 diabetes. Mera: Diabetes Internacional [serie de internet]. 2007 [citado 26 de may 2013]; Septiembre: 18,19. Disponible en: http://indexmedicus.afro.who.int/iah/fulltext/D IJ/nov07/DI\%20Nov\%2007\%2018-19.pdf

18. Figueroa C. Funcionalidad familiar y control glucémico del usuario con diabetes mellitus tipo 2, HNDAC [tesis de bachillerato]. Lima: Universidad César Vallejo; 2012.

19. Jiménez-Benavides A, Gómez SV, AlánisNiño G. Función familiar: Control de diabetes mellitus tipo2. RESPYN [serie de internet]. Feb 2005 [citado 10 de ene 2014]; edición especial (8): [aproximadamente 02 laudas]. Disponible

en http://www.respyn.uanl.mx/especiales/2005/ee -08-2005/documentos 